\title{
Methicillin-resistant Staphylococcus aureus isolates from Iranian restaurant food samples: Panton-Valentine Leukocidin, SCCmec phenotypes and antimicrobial resistance
}

\author{
Reza Ranjbar ${ }^{1}$, Mohammad Hossein Sakhaei Shahreza ${ }^{2}$, Ebrahim Rahimi ${ }^{3}$ and \\ Nematollah Jonaidi-Jafari ${ }^{4 *}$ \\ ${ }^{1}$ Molecular Biology Research Center, Baqiyatallah University of Medical Sciences, Tehran, ${ }^{2}$ Faculty of Veterinary Medicine, \\ Shahrekord Branch, Islamic Azad University, Shahrekord, ${ }^{3}$ Department of Food Hygiene, Faculty of Veterinary Medicine, \\ Shahrekord, ${ }^{4}$ Health Research Center, Baqiyatallah University of Medical Sciences, Tehran, Iran
}

${ }^{\star}$ For correspondence: Email: Jonaidii2000@yahoo.com; Tel: +989123048157

Sent for review: 5 January 2017

Revised accepted: 13 July 2017

\begin{abstract}
Purpose: To assess the distribution of Panton-Valentine Leukocidin, SCCmec types and antimicrobial resistance pattern of methicillin resistant Staphylococcus aureus isolated from restaurant food.

Methods: Five-hundred and eighty food samples were collected and directly transported to the laboratory. Samples were cultured and S. aureus strains were confirmed using biochemical tests. MRSA strains were determined using polymerase chain reaction (PCR)-based amplification of mecA and femA genes. MRSA strains were then subjected to disk diffusion methods.

Results: One-hundred and nineteen out of 580 samples (20.51 \%) were positive for S. aureus. Eightythree out of 119 S. aureus (69.74 \%) were methicillin-resistant. Thirty-nine out of 83 MRSA samples (46.98 \%) harbored PVL gene. Cooked chicken (37\%) had the highest prevalence of S. aureus. Marked seasonality was observed for the prevalence of bacteria. MRSA strains exhibited high resistance against penicillin G $(100 \%)$, tetracycline $(92.77 \%)$, oxacillin (83.13\%) and azithromycin (71.08\%). All MRSA bacteria were resistant to at least 2 antibiotics (100 \%). TetK (80.72\%), linA (67.46 \%), aadA1 $(62.65 \%)$, and msrA (55.42\%) were the most frequently identified resistance genes. SCCmec V (57.83 $\%)$, SCCmec Iva (55.42 \%) and SCCmec IVb (30.12\%) were the most frequent.

Conclusion: Based on the high prevalence of resistant MRSA strains and also high consumption rate of restaurant foods in Iran, it is essential to exercise control over the hygienic conditions of restaurant foods to minimize MRSA strains.
\end{abstract}

Keywords: Methicillin-resistant Staphylococcus aureus (MRSA), Antibiotic resistance, SCCmec types, Panton-Valentine Leukocidin, Restaurant food

Tropical Journal of Pharmaceutical Research is indexed by Science Citation Index (SciSearch), Scopus, International Pharmaceutical Abstract, Chemical Abstracts, Embase, Index Copernicus, EBSCO, African Index Medicus, JournalSeek, Journal Citation Reports/Science Edition, Directory of Open Access Journals (DOAJ), African Journal Online, Bioline International, Open-J-Gate and Pharmacy Abstracts

\section{INTRODUCTION}

Food containing pathogenic agents, causes more than two-hundred diseases al-around the world $[1,2]$. Foodborne diseases cause around 75 million diseases, 330,000 hospitalizations, and
5,000 deaths in the United States annually [3]. Most of these food-borne diseases outbreaks occurred in public places like restaurants. Probable presence of children, elders, sick persons and even immune-suppressive persons in restaurants caused staffs of restaurant to 
seriously considered principles of food hygiene [1-3].

Staphylococcus aureus (S. aureus) is a coccal bacterium that is a member of the Firmicutes, and is commonly originate from the respiratory system and the skin [2-4]. This bacterium is a causative agent of nosocomial infections and foodborne diseases globally [2-4]. Meat is one of the most important food stuffs related to the Staphylococcal foodborne diseases $[4,5]$.

Staphylococcal food-borne and acquired infections are primarily related to the rise of antibiotic resistance $[6,7]$. $S$. aureus has the highest points of resistance against methicillin and other beta-lactam antimicrobial agents $[6,7]$. Prevalence of methicillin-resistant $S$. aureus (MRSA) strains is $50 \%$ in various types of hospital infections [6,7]. Resistance of bacterial strains against antibiotics mainly occurred by presence of antibiotic resistance genes [8].

MecA is a specific gene responsible for occurrence of resistance against methicillin. This geneis carried on a 21- to $67-\mathrm{kb}$ element, the staphylococcal chromosomal cassette mec (SCCmec), which participates at a preserved location in the S. aureus genome [9]. SCCmec elements are typically divided into I, II, III, IV and V types [9]. Type IV is classified to IVa, IVb, IVc and IVd alleles [9]. Data revealed that PantonValentine Leukocidin (PVL) is the most important virulence factor detected in the MRSA strains isolated from the cases of foodborne and clinical infections [10]. Inappropriately, major principles of food hygiene have not been addressed in most Iranian restaurants. Therefore, from the microbiological and epidemiological perspectives, it is important to know the exact hygienic conditions of restaurant foods especially for MRSA which has a global emergence. The current research was done to study the prevalence of MRSA in various types of restaurant foods as well as study the distribution of PVL gene, SCCmec types and antibiotic resistance properties of bacterial isolates.

\section{EXPERIMENTAL}

\section{Ethical considerations}

The study was permitted by the Moral Board of Exploration of the Baqiyatallah University of Medical Sciences, Tehran, Iran (Consent ref no. 110523741). Confirmation of this project and the authorizations related to sampling procedure were obtained from Professors Reza Ranjbar and Ebrahim Rahimi (approval ref no. Med 3932017).

\section{Sample collection and S. aureus identification}

From February 2015 to February 2016, a total of 580 samples were randomly collected from various types of raw and cooked restaurant foods including raw meat $(n=70)$, raw chicken $(n=70)$ and raw fish $(n=70)$ and cooked meat $(n=100)$, cooked chicken $(n=100)$, cooked fish $(n=100)$, and soup $(\mathrm{n}=70)$. Cooked samples were produced by quick-cooking of meat on charcoal (barbecue). Samples were collected under sterile hygienic conditions and were immediately transported to the laboratory at $4{ }^{\circ} \mathrm{C}$. Samples were collected from the various regions of Isfahan province, Iran.

A $25 \mathrm{~g}$ crushed food sample was homogenized using a grinder under aseptic conditions and it was added to $225 \mathrm{~mL}$ of sterile Buffered Peptone Water (Merck, Germany) and incubated at $37^{\circ} \mathrm{C}$ for $24 \mathrm{~h}$. Thereafter, $0.1 \mathrm{~mL}$ of sample was plated onto Baird-Parker agar complemented with egg yolk telluride emulsion and incubated at $37^{\circ} \mathrm{C}$ for 24 to $48 \mathrm{~h}$. Colonies showing characteristic phenotype of $S$. aureus (circular, black, convex colonies surrounded by 2- to $5-\mathrm{mm}$ clear zones) were sub-cultured on $5 \%$ sheep blood agar to isolate single colonies. $S$. aureus was identified through a characteristic hemolysis pattern on sheep blood agar, Gram staining results, catalase reaction (using $0.3 \%$ hydrogen peroxide), mannitol fermentation and coagulase tests.

\section{Identification of methicillin-resistant Staphy- lococcus aureus}

Identification of MRSA strains was done using the PCR-based method. Bacterial strains were sub-cultured in Tryptic Soy Broth (TSB, Merck, Germany) and further incubated for $48 \mathrm{~h}$ at 37 ${ }^{\circ} \mathrm{C}$. DNA was extracted from bacterial colonies using the DNA extraction and purification kit (Fermentas, Germany) according to manufacturer's instruction. The DNA concentration has been determined by measuring absorbance of the sample at $260 \mathrm{~nm}$ using spectrophotometer [11]. Those DNA samples which were concurrently positive for femA and mecA genes were considered as MRSA. For this purpose, PCR method which was introduced by Jonas et al was used [12].

\section{Antimicrobial susceptibility pattern of MRSA}

Pattern of antimicrobial resistance of the $S$. aureus strains was determined using the simple 
disk diffusion assay. Mueller-Hinton agar (Merck, Germany) medium was applied for this goal. Interoperation was done following the guidelines of Clinical and Laboratory Standards Institute [13]. Susceptibility of $S$. aureus isolates were tested against ampicillin (10 u/disk), streptomycin (10 $\mu \mathrm{g} /$ disk), chloramphenicol (30 $\mu \mathrm{g} /$ disk), enrofloxacin ( $5 \mu \mathrm{g} /$ disk), lincomycin $(2 \mu \mathrm{g} /$ disk), cephalothin $(30 \mu \mathrm{g} / \mathrm{disk})$, imipenem (30 u/disk), tetracycline $(30 \mu \mathrm{g} /$ disk $), \quad$ vancomycine (5 $\mu \mathrm{g} /$ disk), ciprofloxacin (5 $\mu \mathrm{g} / \mathrm{disk})$, norfloxacin (30 $\mu \mathrm{g} /$ disk), cotrimoxazole (30 $\mu \mathrm{g} /$ disk), clindamycin (2 $\mathrm{\mu g} /$ disk), trimethoprim-sulfamethoxazole (25 $\mu \mathrm{g} /$ disk), penicillin $\mathrm{G}(10 \mathrm{u} / \mathrm{disk})$, oxacillin (1 $\mu \mathrm{g} /$ disk), erythromycin $(15 \mu \mathrm{g} /$ disk $), \quad$ and azithromycin (15 $\mu \mathrm{g} /$ disk) antibiotic agents (Oxoid, UK). All bacteria were cultured on Mueller-Hinton agar and antibiotic discs were added to media and all were incubated at $37^{\circ} \mathrm{C}$ for $24 \mathrm{~h}$. Diameter of the inhibition zone was measured and interpreted.

\section{Detection of antibiotic-resistance genes, SCCmec types and Panton-Valentine Leukocidin}

Table 1 indicates the list of primers and PCR circumstances used for amplification of antibiotic resistance genes, SCCmec types and PVL gene in the MRSA $[14,15]$. The DNA thermo-cycler (Eppendorf Mastercycler 5330, EppendorfNethel-Hinz GmbH, Hamburg, Germany) were used in all PCR reactions.

Fifteen microliters of the PCR products were subjected to electrophoresis in a $1.5 \%$ agarose gel in 1X TBE buffer at $80 \mathrm{~V}$ for $30 \mathrm{~min}$, stained with SYBR Green (Fermentas, Germany). PCR grade water was used as a negative control and $S$. aureus ATCC 6538 were used as positive control.

\section{Statistical analysis}

Statistical analysis was performed using the SPSS 21.0 software. The chi-square and Fisher's exact tests were performed on obtained data to identify any significant differences for the prevalence of $S$. aureus, PVL genes, SCCmec types and antibiotic resistance properties. Statistical significance was regarded at $p<0.05$.

\section{RESULTS}

\section{Prevalence of S. aureus, MRSA and PVL gene}

Table 2 shows the total prevalence of $S$. aureus, MRSA and also the PVL gene in various types of restaurant food samples. One-hundred and nineteen out of 580 restaurant food samples
$(20.51 \%)$ were positive for S. aureus. Eightythree out of $119 \mathrm{~S}$. aureus strains isolated from restaurant food samples $(69.74 \%)$ were methicillin-resistant. Thirty-nine of 83 MRSA strains (46.98\%) harbored the PVL gene. Cooked chicken (37\%) had the highest prevalence of $S$. aureus, followed by raw chicken $(35.71 \%)$ and cooked meat $(31 \%)$. There was no positive results for the raw fish. Statistically significant difference was seen between the incidence of $S$. aureus and type of food samples $(p<0.05)$ and also between the prevalence of MRSA and type of food samples $(p<0.05)$.

\section{Seasonal distribution of MRSA}

Figure 1 shows the seasonal distribution of MRSA isolated from various types of restaurant food samples. We found that the prevalence of MRSA strains in all types of restaurant food samples were entirely higher in winter seasons than other seasons of the year. Statistically significant difference was seen for the prevalence of MRSA between cold and warm seasons $(P<0.05)$.

\section{Antibiotic resistance of MRSA}

Figure 2 exhibits the incidence of multi-drug resistance in the MRSA strains of restaurant food samples. All of the MRSA strains were resistant to at least 2 antibiotics (100\%), while prevalence of resistance against ten, eleven, twelve, thirteen, fourteen, fifteen and more than fifteen antibiotics were $26.50,19.27,14.45,9.63,7.22$, 3.61 and $1.20 \%$, respectively. Table 3 shows the antibiotic resistance pattern of MRSA strains isolated from various types of restaurant food samples. MRSA strains of our study exhibited the highest levels of resistance against penicillin G $(100 \%)$, tetracycline $(92.77 \%)$, oxacillin (83.13 $\%)$, azithromycin (71.08 \%), trimethoprimsulfamethoxazole (67.46\%), vanco-mycin (61.44 $\%)$ and cotrimoxazole (61.44 \%). Statistically substantial variance was seen for the prevalence of antibiotic resistance between raw and cooked food samples $(p<0.05)$. MRSA strains of cooked chicken samples had the highest and also the most diverse antibiotic resistance pattern.

Table 4 indicates the total frequency of antibiotic resistance genes in the MRSA strains isolated from different types of restaurant food samples. We obtained that tetK $(80.72 \%), \operatorname{lin} A(67.46 \%)$, aadA1 (62.65\%), and $\operatorname{msr} A(55.42 \%)$ were the most routinely identified antibiotic resistance genes in the MRSA strains of restaurant food samples. 
Table 1: Oligonucleotide primers and PCR conditions used for detection of antibiotic resistance genes in MRSA strains isolated from various types of restaurant food

\begin{tabular}{|c|c|c|c|c|}
\hline Target gene & Primer sequence (5'-3') & $\begin{array}{l}\text { PCR product } \\
\text { (bp) }\end{array}$ & PCR programs & PCR volume (50 $\mu \mathrm{L})$ \\
\hline AacA-D & $\begin{array}{l}\text { F: TAATCCAAGAGCAATAAGGGC } \\
\text { R: GCCACACTATCATAACCACTA }\end{array}$ & 227 & \multirow{5}{*}{ 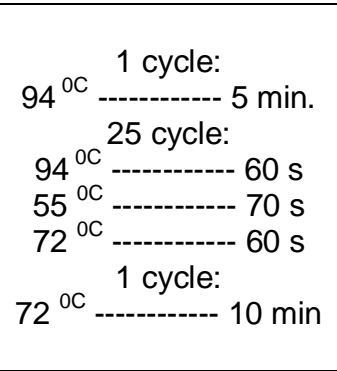 } & \multirow{5}{*}{$\begin{array}{c}5 \mu \mathrm{L} \text { PCR buffer 10X } \\
1.5 \mathrm{mM} \mathrm{Mgcl}_{2} \\
200 \mu \mathrm{M} \text { dNTP (Fermentas) } \\
0.5 \mu \mathrm{M} \text { of each primers F \& R } \\
1.25 \mathrm{U} \text { Taq DNA polymerase } \\
\text { (Fermentas) } \\
2.5 \mu \mathrm{L} \text { DNA template }\end{array}$} \\
\hline ermA & $\begin{array}{l}\text { F: AAGCGGTAAACCCCTCTGA } \\
\text { R: TTCGCAAATCCCTTCTCAAC }\end{array}$ & 190 & & \\
\hline ermC & $\begin{array}{l}\text { F: AATCGTCAATTCCTGCATGT } \\
\text { R: TAATCGTGGAATACGGGTTTG }\end{array}$ & 299 & & \\
\hline tetK & $\begin{array}{l}\text { F: GTAGCGACAATAGGTAATAGT } \\
\text { R: GTAGTGACAATAAACCTCCTA }\end{array}$ & 360 & & \\
\hline vatC & $\begin{array}{l}\text { F: AAAATCGATGGTAAAGGTTGGC } \\
\text { R: AGTTCTGCAGTACCGGATTTGC }\end{array}$ & 467 & & \\
\hline tetM & $\begin{array}{c}\text { F: AGTGGAGCGATTACAGAA } \\
\text { R: CATATGTCCTGGCGTGTCTA }\end{array}$ & 158 & \multirow{2}{*}{ 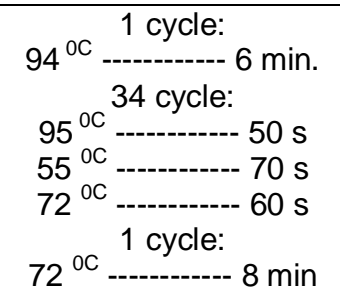 } & \multirow{2}{*}{$\begin{array}{c}5 \mu \mathrm{L} \text { PCR buffer 10X } \\
2 \mathrm{mM} \text { Mgcl }_{2} \\
200 \mu \mathrm{M} \text { dNTP (Fermentas) } \\
0.5 \mu \mathrm{M} \text { of each primers F \& } \mathrm{R} \\
1.5 \mathrm{U} \text { Taq DNA polymerase } \\
\text { (Fermentas) } \\
5 \mu \mathrm{L} \text { DNA template }\end{array}$} \\
\hline vatA & $\begin{array}{l}\text { F: TGGTCCCGGAACAACATTTAT } \\
\text { R: TCCACCGACAATAGAATAGGG }\end{array}$ & 268 & & \\
\hline$m s r A$ & $\begin{array}{c}\text { F: GGCACAATAAGAGTGTTTAAAGG } \\
\text { R: AAGTTATATCATGAATAGATTGTCCTGTT }\end{array}$ & 940 & \multirow{2}{*}{ 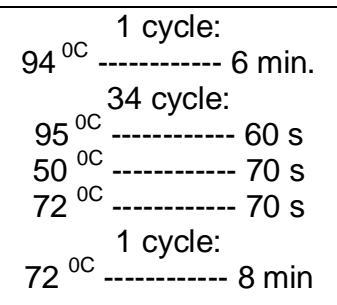 } & \multirow[b]{2}{*}{$\begin{array}{c}5 \mu \mathrm{L} \text { PCR buffer 10X } \\
2 \text { mM Mgcl } \\
150 \mu \mathrm{M} \text { dNTP (Fermentas) } \\
0.75 \mu \mathrm{M} \text { of each primers F \& } \\
1.5 \mathrm{U} \text { Taq DNA polymerase } \\
\text { (Fermentas) } \\
3 \mu \mathrm{L} \text { DNA template }\end{array}$} \\
\hline$m s r B$ & $\begin{array}{c}\text { F: TATGATATCCATAATAATTATCCAATC } \\
\text { R: AAGTTATATCATGAATAGATTGTCCTGTT }\end{array}$ & 595 & & \\
\hline vatB & $\begin{array}{l}\text { F: GCTGCGAATTCAGTTGTTACA } \\
\text { R: CTGACCAATCCCACCATTTTA }\end{array}$ & 136 & 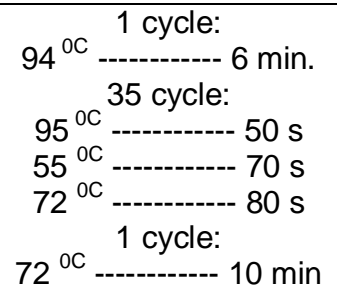 & $\begin{array}{c}5 \mu \mathrm{L} \text { PCR buffer 10X } \\
2 \text { mM Mgcl } \\
150 \mu \mathrm{M} \text { dNTP (Fermentas) } \\
0.75 \mu \mathrm{M} \text { of each primers F \& R } \\
1.5 \mathrm{U} \text { Taq DNA polymerase } \\
\text { (Fermentas) } \\
3 \mu \mathrm{L} \text { DNA template }\end{array}$ \\
\hline
\end{tabular}


Table 1 (continued): Oligonucleotide primers and PCR conditions used for detection of antibiotic resistance genes in MRSA strains isolated from various types of restaurant food

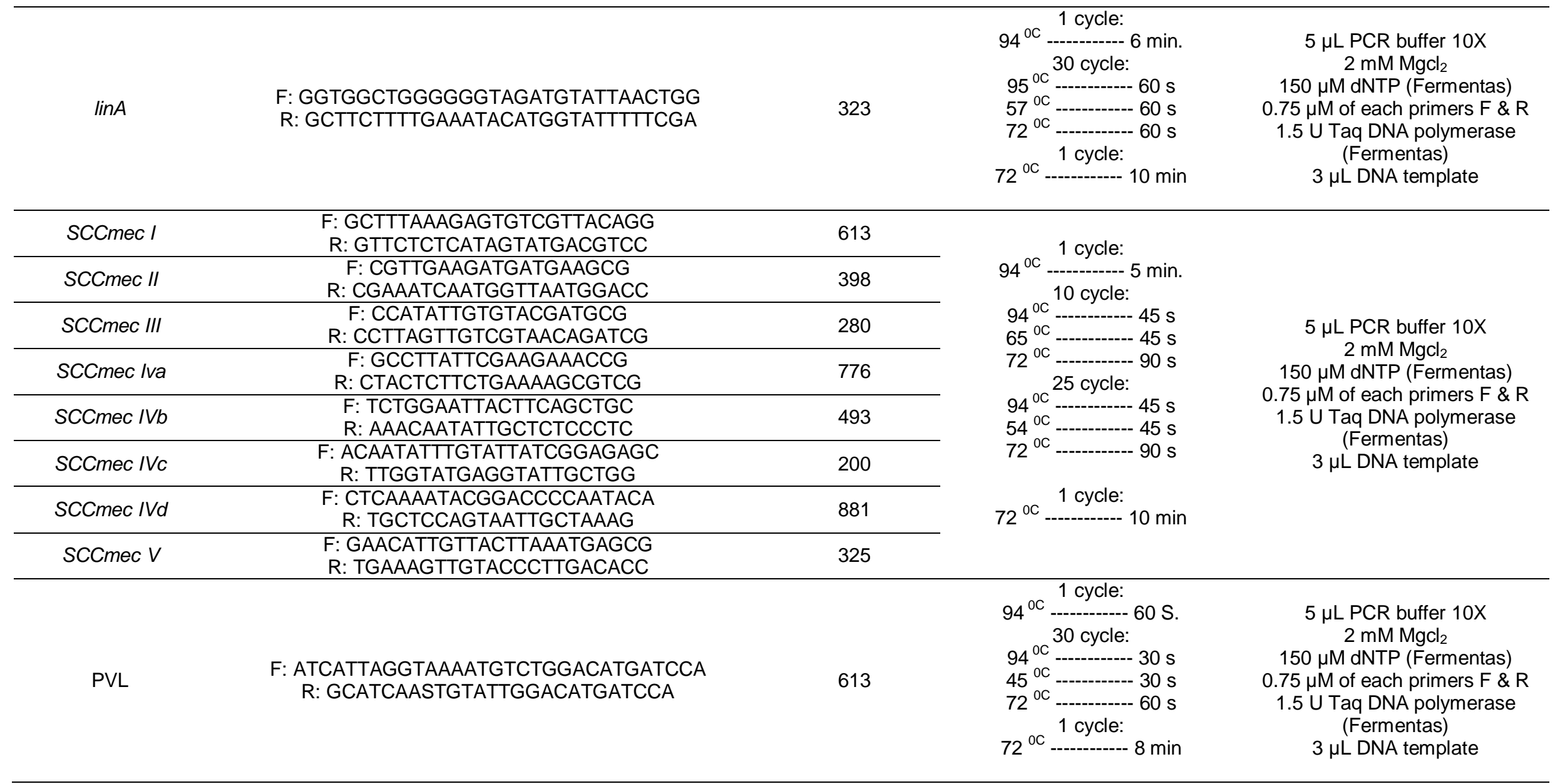


Table 2: Total distribution of $S$. aureus, MRSA and PVL gene in various types of restaurant food samples

\begin{tabular}{lcccc}
\hline Sample type & $\begin{array}{c}\text { No. of samples } \\
\text { collected }\end{array}$ & $\begin{array}{c}\text { No. of S. aureus } \\
\text { strains (\%) }\end{array}$ & $\begin{array}{c}\text { No. of MRSA } \\
\text { strains (\%) }\end{array}$ & $\begin{array}{c}\text { PVL positive } \\
(\%)\end{array}$ \\
\hline Raw meat & 70 & $20(28.57)$ & $10(50)$ & $3(30)$ \\
Raw chicken & 70 & $25(35.71)$ & $13(52)$ & $4(30.765)$ \\
Raw fish & 70 & - & - & - \\
Cooked meat & 100 & $31(31)$ & $25(80.64)$ & $12(48)$ \\
Cooked chicken & 100 & $37(37)$ & $30(81.08)$ & $17(56.66)$ \\
Cooked fish & 100 & $4(4)$ & $3(75)$ & $2(66.66)$ \\
Soup & 70 & $2(2.85)$ & $2(100)$ & $1(50)$ \\
Total & $\mathbf{5 8 0}$ & $\mathbf{1 1 9}(\mathbf{2 0 . 5 1 )}$ & $\mathbf{8 3}(\mathbf{6 9 . 7 4})$ & $\mathbf{3 9 ( 4 6 . 9 8 )}$ \\
\hline
\end{tabular}
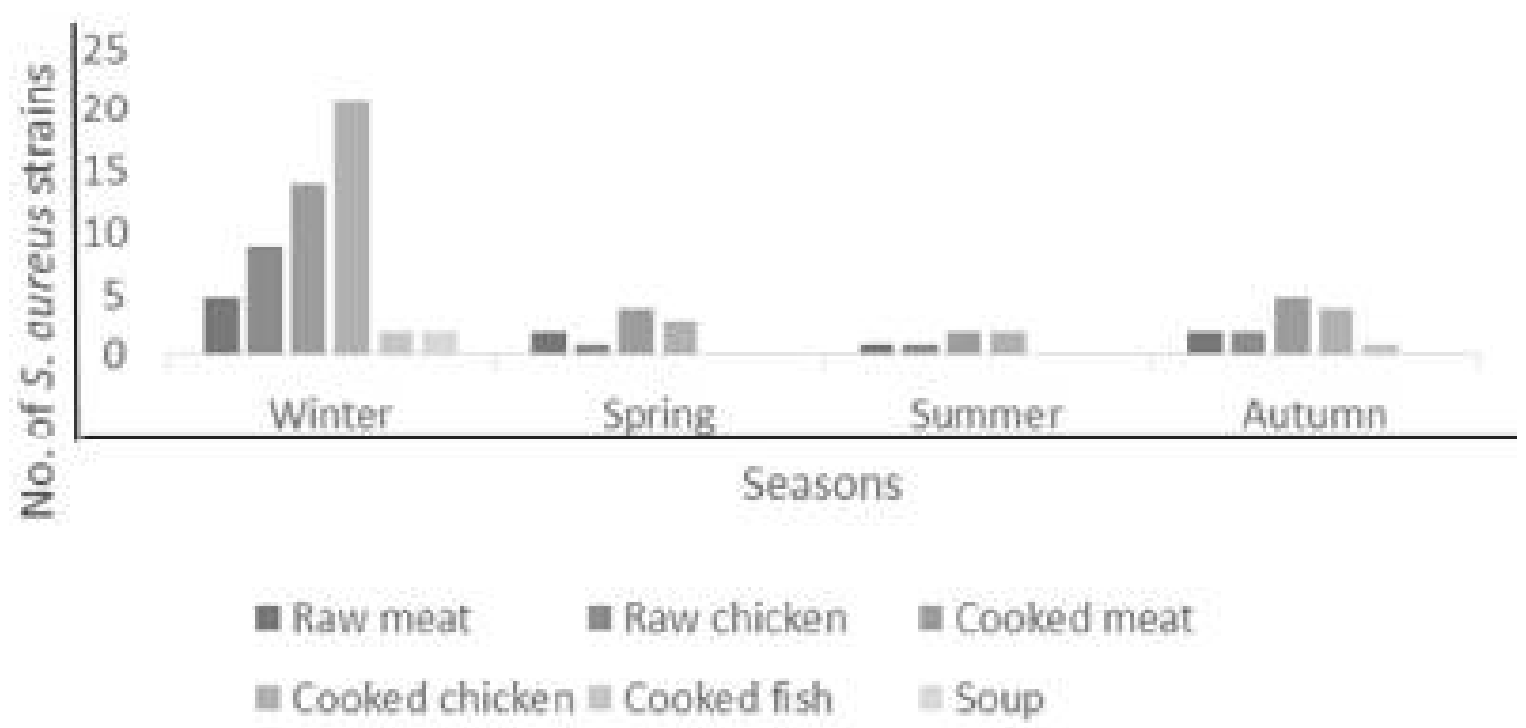

Figure 1: Seasonal distribution of MRSA in various types of restaurant food

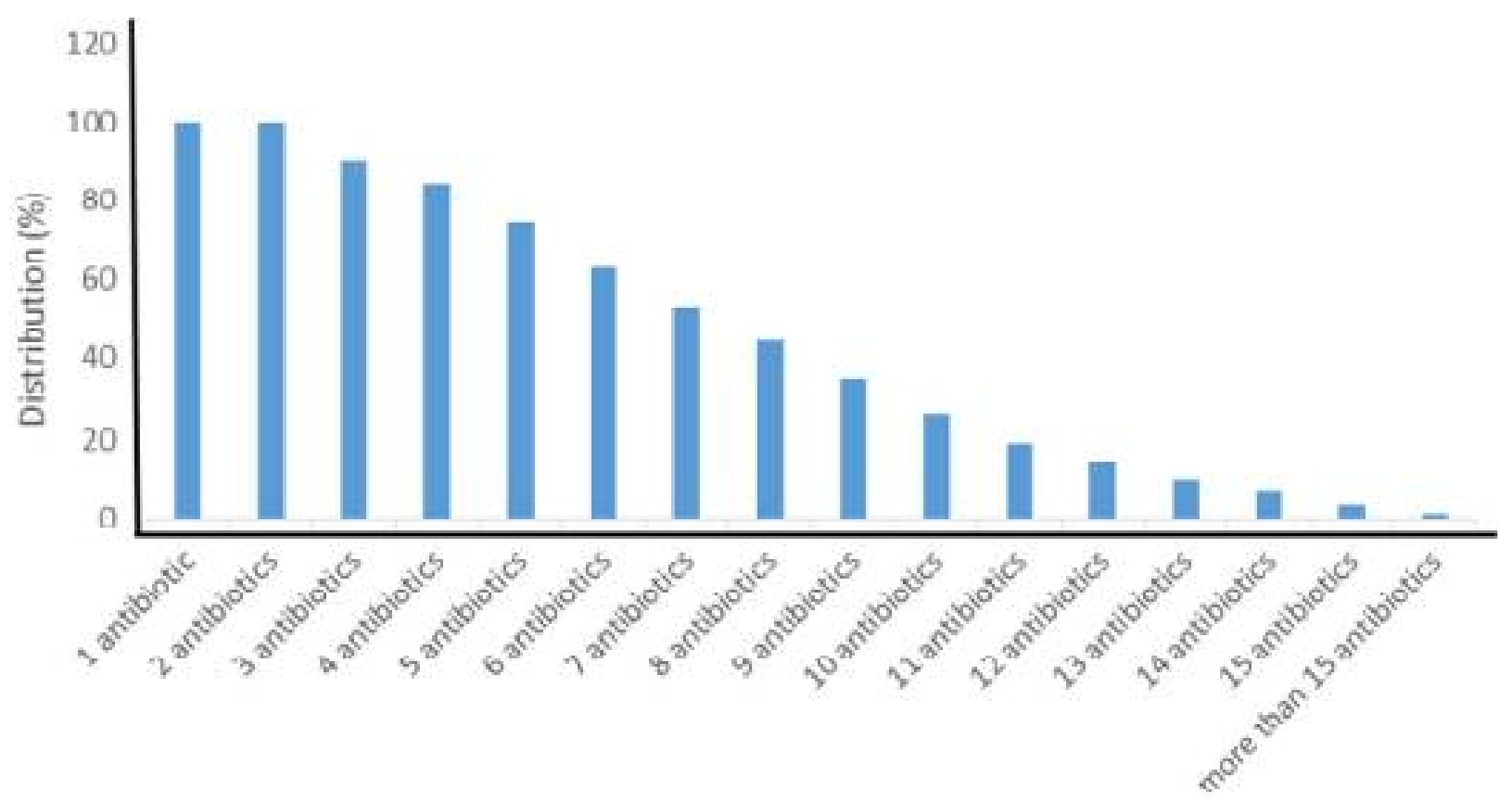

Level of resistance

Figure 2: Total distribution of multi-drug resistance pattern of MRSA strains in restaurant food 
Table 3: Antibiotic resistance pattern of MRSA strains isolated from various types of restaurant food

\begin{tabular}{|c|c|c|c|c|c|c|c|c|c|}
\hline \multirow{2}{*}{$\begin{array}{l}\text { Types of } \\
\text { food } \\
\text { (no. of } \\
\text { MRSA) }\end{array}$} & \multicolumn{9}{|c|}{ Antibiotic resistance pattern (\%) } \\
\hline & A10 & S10 & C30 & Nfx & Lin & Cph & Imp & Tet & Van \\
\hline $\begin{array}{c}\text { Raw } \\
\text { meat } \\
(10)\end{array}$ & $5(50)$ & $6(60)$ & $1(10)$ & $5(50)$ & $5(50)$ & $2(20)$ & - & $8(80)$ & $4(40)$ \\
\hline $\begin{array}{c}\text { Raw } \\
\text { chicken } \\
(13)\end{array}$ & $\begin{array}{c}5 \\
(38.46)\end{array}$ & $\begin{array}{c}6 \\
(46.15)\end{array}$ & $\begin{array}{c}6 \\
(46.15)\end{array}$ & $\begin{array}{c}5 \\
(38.46)\end{array}$ & $\begin{array}{c}5 \\
(38.46)\end{array}$ & $\begin{array}{c}2 \\
(15.38)\end{array}$ & - & $9(90)$ & $\begin{array}{c}5 \\
(38.46)\end{array}$ \\
\hline $\begin{array}{c}\text { Cooked } \\
\text { meat } \\
(25)\end{array}$ & $16(64)$ & $15(60)$ & $1(4)$ & $15(60)$ & $16(64)$ & $8(32)$ & $5(20)$ & $\begin{array}{c}25 \\
(100)\end{array}$ & $17(68)$ \\
\hline $\begin{array}{l}\text { Cooked } \\
\text { chicken } \\
(30)\end{array}$ & $\begin{array}{c}22 \\
(73.33)\end{array}$ & $18(60)$ & $\begin{array}{c}7 \\
(23.33)\end{array}$ & $18(60)$ & $\begin{array}{c}20 \\
(66.66)\end{array}$ & $\begin{array}{c}13 \\
(43.33)\end{array}$ & $9(30)$ & $\begin{array}{c}30 \\
(100)\end{array}$ & $\begin{array}{c}22 \\
(73.33)\end{array}$ \\
\hline $\begin{array}{l}\text { Cooked } \\
\text { fish (3) }\end{array}$ & $\begin{array}{c}1 \\
(33.33)\end{array}$ & - & - & - & $\begin{array}{c}1 \\
(33.33)\end{array}$ & $\begin{array}{c}2 \\
(66.66)\end{array}$ & $\begin{array}{c}1 \\
(33.33)\end{array}$ & $3(100)$ & $\begin{array}{c}2 \\
(66.66)\end{array}$ \\
\hline Soup (2) & - & 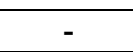 & - & - & - & $1(50)$ & - & $2(100)$ & $1(50)$ \\
\hline $\begin{array}{l}\text { Total } \\
\text { (83) }\end{array}$ & $\begin{array}{c}49 \\
(59.03)\end{array}$ & $\begin{array}{c}45 \\
(54.21)\end{array}$ & $\begin{array}{c}15 \\
(18.07)\end{array}$ & $\begin{array}{c}43 \\
(51.80)\end{array}$ & $\begin{array}{c}47 \\
(56.62)\end{array}$ & $\begin{array}{c}28 \\
(33.73)\end{array}$ & $\begin{array}{c}15 \\
(18.07)\end{array}$ & $\begin{array}{c}77 \\
(92.77)\end{array}$ & $\begin{array}{c}51 \\
(61.44)\end{array}$ \\
\hline
\end{tabular}

\begin{tabular}{|c|c|c|c|c|c|c|c|c|c|}
\hline & \multicolumn{9}{|c|}{ Antibiotic resistance pattern (\%) } \\
\hline & Cip & Nor & Cot & Cln & Trs & Pen & Ox & Ert & $\mathbf{A z}$ \\
\hline $\begin{array}{l}\text { Raw } \\
\text { meat } \\
(10)\end{array}$ & $1(10)$ & $2(20)$ & $4(40)$ & $2(20)$ & $5(50)$ & $\begin{array}{c}10 \\
(100)\end{array}$ & $7(70)$ & $3(30)$ & $6(60)$ \\
\hline $\begin{array}{l}\text { Raw } \\
\text { chicken } \\
\text { (13) }\end{array}$ & $\begin{array}{c}2 \\
(15.38)\end{array}$ & $\begin{array}{c}3 \\
(23.07)\end{array}$ & $\begin{array}{c}5 \\
(38.46)\end{array}$ & $\begin{array}{c}4 \\
(30.76)\end{array}$ & $\begin{array}{c}6 \\
(46.15)\end{array}$ & $\begin{array}{c}10 \\
(100)\end{array}$ & $\begin{array}{c}8 \\
(61.53)\end{array}$ & $\begin{array}{c}4 \\
(30.76)\end{array}$ & $\begin{array}{c}9 \\
(69.23)\end{array}$ \\
\hline $\begin{array}{l}\text { Cooked } \\
\text { meat } \\
\text { (25) }\end{array}$ & $7(28)$ & $10(40)$ & $18(72)$ & $11(44)$ & $19(76)$ & $\begin{array}{c}25 \\
(100)\end{array}$ & $22(88)$ & $13(52)$ & $18(72)$ \\
\hline $\begin{array}{l}\text { Cooked } \\
\text { chicken } \\
\text { (30) }\end{array}$ & $9(30)$ & $12(40)$ & $21(70)$ & $\begin{array}{c}14 \\
(46.66)\end{array}$ & $\begin{array}{c}23 \\
(76.66)\end{array}$ & $\begin{array}{c}30 \\
(100)\end{array}$ & $27(90)$ & $\begin{array}{c}17 \\
(56.66\end{array}$ & $\begin{array}{c}23 \\
(76.66)\end{array}$ \\
\hline $\begin{array}{l}\text { Cooked } \\
\text { fish (3) }\end{array}$ & $\begin{array}{c}1 \\
(33.33)\end{array}$ & $\begin{array}{c}1 \\
(33.33)\end{array}$ & $\begin{array}{c}2 \\
(66.66)\end{array}$ & $\begin{array}{c}1 \\
(33.33)\end{array}$ & $\begin{array}{c}2 \\
(66.66)\end{array}$ & $\begin{array}{c}3 \\
(100)\end{array}$ & $3(100)$ & $\begin{array}{c}1 \\
(33.33)\end{array}$ & $\begin{array}{c}2 \\
(66.66)\end{array}$ \\
\hline $\begin{array}{l}\text { Soup } \\
\text { (2) }\end{array}$ & - & - & $1(50)$ & - & $1(50)$ & $\begin{array}{c}2 \\
(100)\end{array}$ & $2(100)$ & - & $1(50)$ \\
\hline $\begin{array}{l}\text { Total } \\
\text { (83) }\end{array}$ & $\begin{array}{c}20 \\
(24.09)\end{array}$ & $\begin{array}{c}28 \\
(33.73)\end{array}$ & $\begin{array}{c}51 \\
(61.44)\end{array}$ & $\begin{array}{c}32 \\
(38.55)\end{array}$ & $\begin{array}{c}56 \\
(67.46)\end{array}$ & $\begin{array}{c}83 \\
(100)\end{array}$ & $\begin{array}{c}69 \\
(83.13)\end{array}$ & $\begin{array}{c}38 \\
(45.78)\end{array}$ & $\begin{array}{c}59 \\
(71.08)\end{array}$ \\
\hline
\end{tabular}

A10: ampicillin (10 u/disk), S10: streptomycin (10 $\mathrm{\mu g} /$ disk), C30: chloramphenicol (30 $\mu \mathrm{g} /$ disk), Nfx: enrofloxacin (5 $\mathrm{\mu g} / \mathrm{disk}$ ), Lin: lincomycin (2 $\mu \mathrm{g} / \mathrm{disk}$ ), Cph: cephalothin (30 $\mu \mathrm{g} / \mathrm{disk})$, Imp: imipenem (30 u/disk), Tet: tetracycline (30 $\mu \mathrm{g} /$ disk), Van: vancomycine (5 $\mu \mathrm{g} / \mathrm{disk})$, Cip: ciprofloxacin (5 $\mu \mathrm{g} / \mathrm{disk})$, Nor: norfloxacin (30 $\mu \mathrm{g} / \mathrm{disk})$, Cot: cotrimoxazole (30 $\mu \mathrm{g} / \mathrm{disk}), \mathrm{Cln}$ : clindamycin (2 $\mu \mathrm{g} / \mathrm{disk})$, Trs: trimethoprim-sulfamethoxazole (25 $\mu \mathrm{g} / \mathrm{disk})$, Pen:

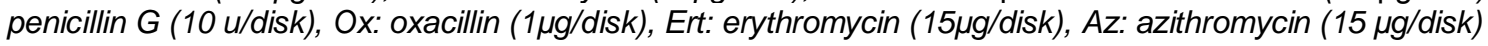

Statistically significant differences were seen between the prevalence of tetk and tetM $(p=$ $0.019)$, ermA and ermC ( $p=0.027)$, vat $A$ and $\operatorname{vatC}(p=0.036)$ and $m s r A$ and $m s r B(p=0.022)$. MRSA strains of cooked chicken samples had the highest and also the most diverse antibiotic resistance genes.

\section{Profile of SCCmec types}

Table 5 represents the total distribution of SCCmec types in the MRSA strains isolated from various types of restaurant food samples. SCCmec V (57.83\%), SCCmec Iva (55.42\%), SCCmec IVb (30.12\%) and SCCmec IVc (20.48 $\%)$ were the most commonly detected types of 
Table 4: Total prevalence of antibiotic resistance genes in MRSA strains isolated from various types of restaurant food

\begin{tabular}{|c|c|c|c|c|c|c|c|}
\hline \multirow{2}{*}{$\begin{array}{l}\text { Sample type (no. of } \\
\text { MRSA strains) }\end{array}$} & \multicolumn{6}{|c|}{ Prevalence of antibiotic resistance genes (\%) } & \multirow[b]{2}{*}{ vatA } \\
\hline & \multirow{2}{*}{$\begin{array}{l}\text { aadA1 } \\
7(70)\end{array}$} & tetK & tetM & \multicolumn{2}{|c|}{ ermA } & ermC & \\
\hline Raw meat (10) & & $7(70)$ & $3(30)$ & \multicolumn{2}{|c|}{$5(50)$} & $3(30)$ & $3(30)$ \\
\hline Raw chicken (13) & $8(61.53)$ & $9(69.23)$ & $2(15.38)$ & \multicolumn{2}{|c|}{$6(46.15)$} & $3(23.07)$ & $6(46.15)$ \\
\hline Cooked meat (25) & $17(68)$ & $20(80)$ & $5(20)$ & \multicolumn{2}{|c|}{$12(48)$} & $6(24)$ & $8(32)$ \\
\hline Cooked chicken (30) & $20(66.66)$ & $26(86.66)$ & $4(13.33)$ & \multicolumn{2}{|c|}{$13(43.33)$} & 7 (23.33) & $11(36.66)$ \\
\hline $\begin{array}{l}\text { Cooked fish (3) } \\
\text { Soup (2) } \\
\text { Total (83) }\end{array}$ & $\begin{array}{c}- \\
- \\
52(62.65)\end{array}$ & $\begin{array}{c}2(100) \\
67(80.72)\end{array}$ & $14(16.86)$ & \multicolumn{2}{|c|}{$\begin{array}{c}2(100) \\
41(49.39)\end{array}$} & $\begin{array}{c}- \\
- \\
18(21.68)\end{array}$ & $\begin{array}{c}2(66.66) \\
2(100) \\
32(38.55)\end{array}$ \\
\hline & \multicolumn{2}{|l|}{ vatB } & \multicolumn{2}{|c|}{ msrA } & \multicolumn{2}{|c|}{ msrB } & $\operatorname{lin} A$ \\
\hline Raw meat (10) & \multicolumn{2}{|l|}{$1(10)$} & \multicolumn{2}{|c|}{$4(40)$} & \multicolumn{2}{|c|}{$3(30)$} & $7(70)$ \\
\hline Raw chicken (13) & \multicolumn{2}{|l|}{$4(30.76)$} & \multicolumn{2}{|c|}{$8(61.53)$} & \multicolumn{2}{|c|}{$4(30.76)$} & $6(46.15)$ \\
\hline Cooked meat (25) & \multicolumn{2}{|l|}{$4(16)$} & \multicolumn{2}{|c|}{$13(52)$} & \multicolumn{2}{|c|}{$7(28)$} & $18(72)$ \\
\hline Cooked chicken (30) & \multicolumn{2}{|l|}{$6(20)$} & \multicolumn{2}{|c|}{$16(53.33)$} & 7( & 3.33) & $22(73.33)$ \\
\hline $\begin{array}{l}\text { Cooked fish (3) } \\
\text { Soup (2) } \\
\text { Total (83) }\end{array}$ & $\begin{array}{c}1(33.33) \\
- \\
16(19.27) \\
\end{array}$ & & $\begin{array}{r}3(1 \\
2(1 \\
46(5\end{array}$ & & 1( & $\begin{array}{l}3.33) \\
- \\
6.50)\end{array}$ & $\begin{array}{c}2(66.66) \\
1(50) \\
56(67.46) \\
\end{array}$ \\
\hline
\end{tabular}

Table 5: Total distribution of SCCmec types in MRSA strains isolated from various types of restaurant food

\begin{tabular}{|c|c|c|c|c|c|c|c|c|}
\hline \multirow{2}{*}{$\begin{array}{l}\text { Type of } \\
\text { sample } \\
\text { (no. of } \\
\text { MRSA } \\
\text { strains) }\end{array}$} & \multicolumn{8}{|c|}{ Prevalence of SCCmec types (\%) } \\
\hline & $\underset{I}{\text { ScCmec }}$ & $\underset{I I}{S c C m e c}$ & $\underset{\text { III }}{\text { scCmec }}$ & 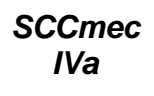 & $\begin{array}{c}\text { SCCmec } \\
I V b\end{array}$ & $\begin{array}{c}\text { ScCmec } \\
\text { IVc }\end{array}$ & $\begin{array}{c}\text { SCCmec } \\
I V d\end{array}$ & $\underset{V}{S c C m e c}$ \\
\hline $\begin{array}{l}\text { Raw meat } \\
(10)\end{array}$ & $1(10)$ & - & $1(10)$ & $4(40)$ & $2(20)$ & $2(20)$ & - & $4(40)$ \\
\hline $\begin{array}{l}\text { Raw } \\
\text { chicken } \\
\text { (13) }\end{array}$ & $1(7.69)$ & - & $1(7.69)$ & $6(46.15)$ & $4(30.76)$ & $3(23.07)$ & $1(7.69)$ & $7(53.84)$ \\
\hline $\begin{array}{l}\text { Cooked } \\
\text { meat (25) }\end{array}$ & $2(8)$ & $2(8)$ & $3(12)$ & $14(56)$ & $7(28)$ & $5(20)$ & $2(8)$ & $15(60)$ \\
\hline $\begin{array}{l}\text { Cooked } \\
\text { chicken } \\
(30)\end{array}$ & $3(10)$ & $2(6.66)$ & $3(10)$ & $18(60)$ & $\begin{array}{c}10 \\
(33.33)\end{array}$ & $6(20)$ & 4 (13.33) & $18(60)$ \\
\hline $\begin{array}{l}\text { Cooked } \\
\text { fish (3) }\end{array}$ & - & - & $1(33.33)$ & $2(66.66)$ & $1(33.33)$ & $1(33.33)$ & - & $2(66.66)$ \\
\hline Soup (2) & - & - & - & $2(100)$ & $1(50)$ & - & - & $2(100)$ \\
\hline Total (83) & $7(8.43)$ & 4 (4.81) & $9(10.84)$ & $\begin{array}{c}46 \\
(55.42)\end{array}$ & $\begin{array}{c}25 \\
(30.12)\end{array}$ & $\begin{array}{c}17 \\
(20.48)\end{array}$ & $7(8.43)$ & $\begin{array}{c}48 \\
(57.83)\end{array}$ \\
\hline
\end{tabular}

SCCmec. Prevalence of SCCmec II, SCCmec I and SCCmec IVd were low. Statistically significant difference was seen for the prevalence of SCCmec types between raw and cooked food samples $(p<0.05)$. MRSA strains of cooked chicken and cooked meat samples had the highest and also the most diverse SCCmec types.

\section{DISCUSSION}

The results of the present investigation showed that resistant and virulent strains of MRSA had a high prevalence, antibiotic resistance and pathogenicity in restaurant food samples. As far as we know, this is the first prevalence report of the MRSA and their antibiotic resistance properties in the restaurant food samples in Iran. We found that $20.51 \%$ of food samples were contaminated with $S$. aureus and among the bacteria isolates $69.74 \%$ were MRSA. High prevalence of MRSA and also the PVL gene $(46.98 \%)$ showed an important public health problem regarding the Iranian restaurants. Using from low quality ingredients, high prescription of antimicrobial agents in veterinary and medicine fields, nonconformity of individual and public hygiene in kitchen and also staffs of kitchens in 
restaurants and lack of adequate time and temperature for cooking of foods are some key factors which may cause high prevalence of MRSA in restaurant food samples of our investigation.

Low prevalence of MRSA in fish meat samples of our investigation may be due, in part, to the presence of specific primary bacterial flora in fish and low ability of $S$. aureus to compete with them for survival. Higher prevalence of MRSA in chicken meat samples may be due, in part, to the high prescription of human-based antibiotics and especially methicillin in the poultry farms in Iran. In addition, higher temperature of poultries body caused the destruction of weak bacteria and survival of more resistant bacteria like $S$. aureus. Another important reason could be the fact that veterinarian used various types of antibiotics in poultry farms as a therapeutic agent and also growth factor. Therefore, other pathogenic bacteria cannot survive well and in this situation $S$. aureus which has the highest levels of resistance can survive without any competitor.

Cooked food had the higher prevalence of $S$. aureus, MRSA and the PVL gene than raw foods. The main reason for this finding in addition to cross-contamination from kitchen staff and environment during the production process into the food samples. Unfortunately, chiefs do not use high enough and adequate temperature for cooking of barbecues. Therefore, the central parts of barbecue samples are not cooked well. In addition, new strains of $S$. aureus can transmit from the infected staff of the kitchens and especially their skin infections and respiratory secretions (which are considered as a main sources of $S$. aureus) to cooked foods which are free from any competitor bacteria. Therefore, MRSA strains can survive without any rival.

Indiscriminate, unauthorized and irregular prescription of antibiotics in veterinary and medical fields caused occurrence of high antibiotic resistance in the MRSA strains of our study. We found that more than $70 \%$ of MRSA strains of our study were resistant to several antibiotics, together. We found that resistance of MRSA strains of cooked samples against human-based antibiotics was completely higher than animal-based antibiotics which can indirectly established the transmission of MRSA strains from the chef of the kitchen and also kitchen staff to food stuffs. In addition, MRSA strains of restaurant food samples and especially cooked samples harbored the high levels of resistance against human-based antibiotics such as cephalothin, imipenem, ciprofloxacin, norfloxacin, cotrimoxazole, clindamycin, trimethoprim-sulfamethoxazole, oxacillin and azithromycin which can indirectly confirm their anthropogenic origin. The prevalence of antibiotic resistance genes and especially those that encode resistance against human-based antibiotics were also higher among MRSA strains isolated from cooked restaurant food samples which also can indirectly confirm the broadcast of anthropogenic MRSA strains possibly from staffs of restaurant kitchens to foods after cooking process.

Several studies have conducted in this field. For instance Puah et al [16] reported that $26 \%$ of restaurant food samples in Malaysia were infected with $S$. aureus which was higher than our results. They showed that $28.80 \%$ of bacterial strains were resistant against tetracycline and the prevalence of multi-drug resistance was $3.80 \%$ which was lower than our findings; (92.77\% resistance against tetracycline and more than $70 \%$ of strains were resistant against multiple antibiotics). Udo et al [17] showed that $S$. aureus had a high prevalence in the restaurant food samples of Kuwait. They reported that $S$. aureus strains were resistant to penicillin G (82.0\%), tetracycline (19.0\%), erythromycin (2.5\%), clindamycin (2.0\%), trimethoprim (7.5\%), kanamycin (2.5\%), streptomycin (1.5\%), ciprofloxacin (1.5\%), fusidic acid (1.0\%) and cadmium acetate $(68.0 \%)$ which was similar to our findings.

Occurrence of resistance against chloramphenicol in the MRSA strains of our examination were $18.07 \%$ which was completely higher in chicken-based samples. Chloramphenicol is a restricted antibiotic. The high prevalence of resistance against chloramphenicol displayed its unequal and illegal use in veterinary treatment and especially in the field of poultry in Iran. Therefore, antibiotic resistance will occur against this antibiotic especially in poultry and chicken samples. There were no additional chloramphenicol-resistant MRSA strains in cooked samples compared to the raw foods which showed that all of these strains had a primary and/or animal-based origin. High prevalence of resistance against chloramphenicol was seen in studies conducted by Momtaz et al [18], Dehkordi et al [19], and Osman et al [20].

Gundogan et al [21] reported that the $S$. aureus strains of food samples had high levels of resistance against penicillin G (53.8\%), while the levels of resistance against erythromycin was low $(7.5 \%)$ which was in harmony with our findings. They showed that all of the $S$. aureus strains were susceptible to vancomycin, sulbactam- 
ampicillin, ciprofloxacin and cefaperazonesulbactam which was in contrast with our results.

Another similar study [17] revealed that the prevalence of resistance of $S$. aureus strains of food samples against penicillin G, tetracycline, erythromycin, clindamycin, trimethoprim, kanamycin, streptomycin and ciprofloxacin were 82.0, 19.0, 2.5, 2.0, 7.5, 2.5, 1.5 and $1.5 \%$, respectively.

We found that $46.98 \%$ of MRSA strains of our study harbored the PVL gene. This gene encodes one of the major exotoxins of the $S$. aureus. High prevalence of this gene showed an important public health problem facing Iranian restaurant foods. Previously published data reported that majority of PVL positive strains of $S$. aureus were accompanying with skin and soft tissue infections [21]. Therefore, PVL positive strains of the $S$. aureus isolates of our investigation maybe transmitted from the handmanipulation of staff into the foods. Absence of the PVL gene in the cases of food poisoning has been reported previously [22].

Additional portion of our inquiry was attentive on the distribution of SCCmec types in MRSA strains of various types of food samples. We found that the distribution of SCCmec I, SCCmec II, SCCmec III, ScCmec IVa, ScCmec IVb, SCCmec IVc, SCCmec IVd and SCCmec $V$ in various types of food samples were $8.43,4.81$, $10.84,55.42,30.12,20.48,8.43$ and $57.83 \%$, respectively. Jackson et al [23] reported similar results for the high prevalence of SCCmec IV in retail meat samples. They reported that all of the MRSA strains of beef and pork samples were positive for SCCmec IV which was higher than our findings. In a study which was conducted by Vossenkuhl et al [24] most of the MRSA strains of turkey meat samples carried SCCmec V (58.1 - $71.9 \%)$ followed by type IVa (19 - $27.0 \%)$ which was similar to our findings. In addition to the results of our study and that of Vossenkuhl et al [24], Zhang et al [15] reported high prevalence of SCCmec III in their food samples. High prevalence of SCCmec IVa and V were also detected in the retail meat samples of Bhargava et al [25].

\section{CONCLUSION}

As far as we know, this is the first prevalence report of the molecular characterization and antimicrobial resistance properties of MRSA strains isolated from restaurant food samples in Iran. An overall incidence of $20.51 \%$ and a relatively high prevalence of $\mathrm{PVL}$ gene, $\mathrm{SCCmec}$ types, antibiotic resistance genes as well as the multi-drug resistance pattern of $S$. aureus show a possible public health threat from Iranian restaurant food. Each MRSA isolate, regardless of their origin, harbor at least one of the main antibiotic resistance genes, indicating their pathogenicity, which must be considered a serious health hazard. Standard hygiene protocols and control measures need to be instituted to mitigate serial health hazards.

\section{DECLARATIONS}

\section{Acknowledgement}

The authors would like to thank Drs Hassan Momtaz and Manouchehr Momeni at Islamic Azad University for their technical support.

\section{Conflict of Interest}

No conflict of interest associated with this work.

\section{Contribution of Authors}

The authors declare that this work was done by the authors named in this article and all liabilities pertaining to claims relating to the content of this article will be borne by them.

\section{Open Access}

This is an Open Access article that uses a funding model which does not charge readers or their institutions for access and distributed under the terms of the Creative Commons Attribution License (http://creativecommons.org/licenses/by/ 4.0) and the Budapest Open Access Initiative (http://www.budapestopenaccessinitiative.org/rea d), which permit unrestricted use, distribution, and reproduction in any medium, provided the original work is properly credited.

\section{REFERENCES}

1. Gould $L H$, Walsh KA, Vieira AR, Herman K, Williams IT, Hall AJ, Cole D. Surveillance for foodborne disease outbreaks-United States, 1998-2008. MMWR Surveill Summ 2013; 62: 1-34.

2. Havelaar AH, Kirk MD, Torgerson PR, Gibb HJ, Hald T, Lake RJ, Praet N, Bellinger DC, de Silva NR, Gargouri $N$, et al. World health organization global estimates and regional comparisons of the burden of foodborne disease in 2010. Plos Med 2015; 12: e1001923.

3. Scallan E, Hoekstra RM, Angulo FJ, Tauxe RV, Widdowson M-A, Roy SL, Jones JL, Griffin PM. Foodborne illness acquired in the united states-major pathogens. Emerg Infect Dis 2011; 17:7-15. 
4. Beneke B, Klees S, Stührenberg B, Fetsch $A$, Kraushaar $B$, Tenhagen BA. Prevalence of methicillin-resistant Staphylococcus aureus in a fresh meat pork production chain. J Food Prot 2011; 74: 126-129.

5. Hanson BM, Dressler AE, Harper AL, Scheibel RP, Wardyn SE, Roberts LK, Kroeger JS, Smith TC. Prevalence of Staphylococcus aureus and methicillinresistant Staphylococcus aureus (MRSA) on retail meat in lowa. J Infect Publ Health 2011; 4: 169-174.

6. Johnson AP. Methicillin-resistant Staphylococcus aureus. the European landscape. J Antimicrob Chemother 2011; 66: 43-48.

7. Morell EA, Balkin DM. Methicillin-resistant Staphylococcus aureus: a pervasive pathogen highlights the need for new antimicrobial development. Yale $\mathrm{J}$ Biol Med 2010; 83: 223-233.

8. Argudín MA, Mendoza MC, Gonzalez-Hevia MA, Bances $M$, Guerra B, Rodicio MR. Genotypes, exotoxin gene content, and antimicrobial resistance of Staphylococcus aureus strains recovered from foods and food handlers. Appl Environ Microbiol 2012; 78: 2930-2935.

9. Ferreira FA, Souza RR, de Sousa Moraes B, de Amorim Ferreira AM. Americo MA, Fracalanzza SE, Dos Santos Silva Couceiro JN, Sa Figueiredo AM. Impact of agr dysfunction on virulence profiles and infections associated with a novel methicillin-resistant Staphylococcus aureus (MRSA) variant of the lineage ST1-SCCmec IV. BMC Microbiol 2013; 27: 13-93.

10. Shrestha B, Singh W, Raj VS, Pokhrel BM, Mohapatra TM. High Prevalence of Panton-Valentine Leukocidin (PVL) Genes in Nosocomial-Acquired Staphylococcus aureus Isolated from Tertiary Care Hospitals in Nepal. BioMed Res Int 2014; 2014:1-7.

11. Sambrook J, Russell D. Molecular cloning, a laboratory manual. New York: Cold Spring Harbor Laboratory; 2001.

12. Jonas D, Speck M, Daschner FD, Grundmann H. Rapid PCR-Based Identification of Methicillin-Resistant Staphylococcus aureus from Screening Swabs. J Clin Microbiol 2002; 40:1821-1823.

13. Clinical and Laboratory Standards Institute (CLSI). Performance standards for antimicrobial susceptibility testing. Twenty-second informational supplement M100 S21. Wayne Pa; 2012.

14. Lina G, Quaglia A, Reverdy M, Leclercq R, Vandenesch $F$, Etienne J. Distribution of genes encoding resistance to macrolides, lincosamides, and streptogramins among Staphylococci. Antimicrob Agent Chemother 1999; 43 : 1062-1066.

15. Zhang K, McClure J, Elsayed S, Louie T, Conly J. Novel multiplex PCR assay for characterization and sub typing of staphylococcal cassette chromosome mec types I to $V$ in methicillin-resistant Staphylococcus aureus. J Clin Microbiol 2005; 43: 5026-5033.

16. Puah SM, Chua KH, Tan JA. Virulence Factors and Antibiotic Susceptibility of Staphylococcus aureus Isolates in Ready-to-Eat Foods: Detection of S. aureus Contamination and a High Prevalence of Virulence Genes. Int J Environ Res Publ Health 2016; 13: 199.

17. Udo EE, Al-Mufti S, Albert MJ. The prevalence of antimicrobial resistance and carriage of virulence genes in Staphylococcus aureus isolated from food handlers in Kuwait City restaurants. BMC Res Notes 2009; 2: 108.

18. Momtaz H, Farzan R, Rahimi E, Safarpoor Dehkordi F, Souod N. Molecular characterization of shiga toxinproducing Escherichia coli isolated from ruminant and donkey raw milk samples and traditional dairy products in Iran. Sci World J 2012; 2012: 231342.

19. Dehkordi FS, Yazdani F, Mozafari J, Valizadeh Y. Virulence factors, serogroups and antimicrobial resistance properties of escherichia coli strains in fermented dairy products. BMC Res Notes 2014; 7: 1.

20. Osman KM, Amer AM, Badr JM, Helmy NM, Elhelw RA, Orabi A, Bakry M, Saad AS. Antimicrobial Resistance, Biofilm Formation and mecA Characterization of Methicillin-Susceptible S. aureus and Non-S. aureus of Beef Meat Origin in Egypt. Front Microbiol 2016; 7: 222.

21. Gundogan N, Citak S, Yucel N, Devren A. A note on the incidence and antibiotic resistance of Staphylococcus aureus isolated from meat and chicken samples. Meat Sci 2005; 69: 807-810.

22. Holmes A, Ganner M, McGuane S, Pitt TL, Cookson BD, Kearns AM. Staphylococcus aureus Isolates Carrying Panton-Valentine Leucocidin Genes in England and Wales: Frequency, Characterization, and Association with Clinical Disease. J Clin Microbiol 2005; 43: $2384-$ 2390.

23. Jackson CR, Davis JA, Barrett JB. Prevalence and Characterization of Methicillin-Resistant Staphylococcus aureus Isolates from Retail Meat and Humans in Georgia. J Clin Microbiol 2013; 51: 1199-1207.

24. Vossenkuhl B, Brandt J, Fetsch A, Kasbohrer A, Kraushaar B. Alt K, Tenhagen BA. Comparison of spa Types, SCCmec Types and Antimicrobial Resistance Profiles of MRSA Isolated from Turkeys at Farm, Slaughter and from Retail Meat Indicates Transmission along the Production Chain. Plos One 2014; 9: e96308.

25. Bhargava $K$, Wang $X$, Donabedian $S$, Zervos $M$, da Rocha L, Zhang Y. Methicillin-Resistant Staphylococcus aureus in Retail Meat, Detroit, Michigan, USA. Emerg Infect Dis 2011; 17: 1135-1137. 